\title{
Reactions of the Autonomic Nervous System of Healthy Male Humans on the Natural and Simulated Conditions of the Geomagnetic Field
}

\author{
Ketevan Janashia ${ }^{1,}$, Levan Tvildiani ${ }^{1}$, Tamar Tsibadze ${ }^{1}$, Nikoloz Invia ${ }^{2}$, Vasili Kukhianidze ${ }^{3}$, \\ George Ramishvili ${ }^{3}$ \\ ${ }^{1}$ Central Scientific Research Laboratory, Aieti Medical school, David Tvildiani Medical University, Tbilisi, Georgia \\ ${ }^{2}$ Department of Biomedical Engineering, Faculty of Informatics and Control Systems, Georgian Technical University, Tbilisi, Georgia \\ ${ }^{3}$ Solar Physics Group, School of Natural Sciences and Medicine, Ilia State University, Tbilisi, Georgia
}

\author{
Email address: \\ k.janashia@dtmu.edu.ge (K. Janashia) \\ ${ }^{*}$ Corresponding author
}

\section{To cite this article:}

Ketevan Janashia, Levan Tvildiani, Tamar Tsibadze, Nikoloz Invia, Vasili Kukhianidze, George Ramishvili. Reactions of the Autonomic Nervous System of Healthy Male Humans on the Natural and Simulated Conditions of the Geomagnetic Field. American Journal of Clinical and Experimental Medicine. Vol. 8, No. 4, 2020, pp. 63-70. doi: 10.11648/j.ajcem.20200804.12

Received: June 22, 2020; Accepted: July 13, 2020; Published: August 4, 2020

\begin{abstract}
Background: The geomagnetic environment is very sensitive to changes in "Space weather" (SW) and its manifestations on the Earth. The human body is adapted evolutionarily to the slight alterations of the geomagnetic field (GMF). Objective: The aim of this work is to test the hypothesis on whether different levels of GMF causing specific stress-reactions in humans' autonomic nervous system (ANS) depending on the levels of GMF. Subjects \& method: This is a randomized control study, in which took part $\mathrm{n}=62,18-24$ years old healthy male volunteers. We measured their ANS responses via heart rate variability (HRV) and stress index (SI) to compare them with the K index of GMF (the magnitude of GMF during geomagnetic storms (GMS)). Experiments were performed as in natural as well as in the lab conditions by simulation of different geomagnetic activity (GMA) using the pilot device of GMS compensation/simulation. Results: In comparison with quiet magnetic days $(\mathrm{K}=1-3)$, the initial values of HRV significantly shifted towards intensification of the sympathetic part (SP) of the ANS during days of GMSs (K=5-7). Significantly shifts in both parts of ANS (sympathetic/parasympathetic) were observed during short-term exposure to simulated GMSs $(K=7$, the magnetic induction $B=200 \mathrm{nT})$ in comparison with conditions during compensated GMSs $(\mathrm{K}=0, \mathrm{~B}=0-5 \mathrm{nT})$. Conclusion: The results indicate an intensification of the ANS as a stress reaction in healthy humans when it is exposed to high levels of GMF in both natural or simulated conditions, however, the initial regulation types of the ANS (balanced/parasympathetic) results in different dynamics in its variation as a function of the GMF level.

Keywords: Heart Rate Variability, Autonomic Nervous System, Sensitive Reactions, Geomagnetic Storms,
\end{abstract} Geomagnetic Storms Compensation/Simulation Device

\section{Introduction}

Solar-induced disturbances of Earth's magnetosphere results in geomagnetic field (GMF) variations. The fundamental difficulty when studying the potential effects of GMF on the human body, compared with other anthropogenic sources, is that the field strengths involved are very small, the GMF varies by a few $\mathrm{nT}$ with the frequencies of less than a hundred Hertz. However, many authors have noticed significant health effects associated with changes in solar-induced geomagnetic activity (GMA). A key scientific question still remains: how paradoxically small parameters of GMF can cause the observed effects and how to detect and evaluate the human response to GMA?

The mechanisms involved in the sensitivity of biological objects to GMF are not yet clearly understood. There are several assumptions and theories that the GMF effects can occur through the magnetoreception mechanisms based on magnetic nanoparticles, cryptochromes, long-living radical 
pairs, liquid water states of yet unknown origin $[1,2]$, or via the modulation of the rate of synthesis of melatonin/serotonin which via receptors influences on the circadian activity of the metabolism including body temperature, blood pressure, heartbeat, peripheral blood flow, respiratory activity, sleep-wake cycle, reaction times, hormone levels and etc. [3, 4]. According to modern conceptions, based on the quantum mechanical resonance theory [5], solar-induced GMF's periodical and non-periodical activities such as geomagnetic storms (GMSs) are correspondingly the synchronizer/desynchronizer factors of resonance modulation for the endogenous biological rhythms of a living organism [6, 7]. Particularly characteristic spectral frequencies of GMF oscillations are often similar/synchronous to the oscillations of major physiological processes, which involves "non-linear resonant absorption" of ultra-low frequency (ULF) oscillating signals in the same frequency range. The human cardiovascular system can be affected, due to the increase in field-line resonances probably by the overlapping of GMF oscillations with the rhythms of the autonomic nervous system (ANS).

Previous studies demonstrated that some spectral indicators of heart rate in healthy subjects coincided with the oscillations of GMF in the dominant mode of frequency range 0.5-3.0 $\mathrm{mHz}[8]$ and with the first five modes of natural ULF signal, Schumann Resonance signal (0-35 Hz) [9-11]. Therefore, during GMSs when the magnitude of GMF sharply increases up to 350-1200nT (http://www.geomag.bgs.ac.uk/education/earthmag.html\#_To c2075560), GMS as a desynchronizer factor breaks the regular, synchronous, circadian rhythm of the vital processes resulting in alterations in hormones balance, blood pressure, immune system, cardiac and neurological processes [12,13] and could be a serious risk to the magneto sensitive people on the Earth and near-Earth [14-16]. Attempts were made to detect and evaluate the effects of GMA on the biological subjects, the number of studies revealing significant associations between GMSs and decreased [12, 17-19] or increased levels of heart rate variability (HRV) with different levels of stress-reactions $[20,21]$. This relates to the idea that different dynamics in HRV could be explained to a different ANS response, which can be different in a particular individual depending on a self-character of ANS regulation type (sympathetic/ balanced/parasympathetic).

$\mathrm{HRV}$ is the naturally occurring change in the time intervals between adjacent pairs of heartbeats reflecting the functional status of interdependent regulatory systems of the human body to adapt to the environmental and psychological challenges [22], HRV permits one to determine the stress level, which precedes the illness [23].

The parasympathetic and sympathetic parts (PP and SP) of the ANS show different responses to stress. The parasympathetic influence prevails at relaxation and the sympathetic factor is dominant under stress, so the ratio of the spectral power of the appropriate frequency ranges (LH/HF) could serve as a quantitative measure of the activity level of the two parts of the ANS [24, 25], where an increase in LF/HF is assumed to reflect a shift to "sympathetic dominance" and a decrease corresponds to "parasympathetic dominance," although there are some opposite assumptions regarding $\mathrm{LF} / \mathrm{HF}$, some authors have noted, that this ratio does not accurately measure the cardiac sympatho-vagal balance [2627]. There are also assumptions that reduced HRV may correlate with disease and mortality because it reflects reduced regulatory capacity and ability to adaptively respond to physiological challenges [28].

Building on cited results the objective of our current study was to detect and evaluate sensitive reactions in humans as a function of GMF perturbations in both: during natural conditions and during simulation of different GMA levels in the experimental room enabling to measure the effects of the direct impact of GMS, while eliminating other low frequency environmental magnetic impacts. In both cases, the evaluation criteria were being the values of the ANS reactions on the different conditions of GMF.

\section{Materials and Experiments}

The study was performed at a middle latitude $\left(41^{\circ} 41^{\prime} 38^{\prime \prime} \mathrm{N}\right)$ in Tbilisi, Georgia. The data on GMA was obtained via the Internet (201504AK.txt - 201507AK.txt, U.S. Dept. of Commerce, NOAA, Space Weather Prediction Center). The geomagnetic K-index [29] was used to characterize the magnitude of the GMS, where days with $\mathrm{K} \leq 2$ were ascribed to magnetically quiet periods and those with $\mathrm{K} \geq 5$ as those when GMSs occurred. In this study, we used a pilot GMSs magneto-active compensation/simulation device designed by Invia et al. [30, 31]. For modeling of different GMA, required data was provided by the magnetometer (FGM3D/100 with a working frequency of $10 \mathrm{~Hz}$ ) that is located in the village of Tskhvarichamia, near Tbilisi, where there is a minimum level of environmental magnetic interference present. The obtained magnetometer recordings were saved in a dataset and reprocessed for GMS simulations [32].

All stages of experiments as in natural as well as in simulated conditions were done in the special created experimental room at central scientific research laboratory of David Tvildiani Medical University.

In experiments took part $\mathrm{n}=62$ (from 102 initially), 18-24 years old, healthy male volunteers (students of DTMU). In this paper, the measurements of ANS responses to different GMA via comparison of the heart rate variability (HRV) indices and stress index (SI) with $\mathrm{K}$-index of GMF are presented and discussed. The HRV measurements were done according to the appropriate methodical guidelines and recommendations [33, 34], using the ArguSys++ Holter monitoring system (www.innomed.hu). SI was calculated from standard parameters of the rhythmo gram [35] using pulse oximeter Contec CMS50E. The research was carried out as a single-blinded randomized control study, using block and within-subject design. A prerequisite precondition for the test volunteers was to avoid any negative influences, resulting from emotional and physical excitation, heavy nutrition, alcohol, etc., within 3 days before recordings. Measurements were done after the breakfast (at least by 1.5-2 hours), with a 
constant temperature ranging within the interval $20-22^{\circ} \mathrm{C}$ in the room. A period of 20 minutes for adaptation of volunteers to local environmental conditions preceded measurements. HRV recordings were done in a supine position with quiet breathing.

\subsection{Experiment 1 - Natural Conditions}

Experiments were performed on a control group (Group 1), that was divided into two subgroups, regarding their initial autonomic regulation types, subgroup $1 \mathrm{~A}$ included volunteers with parasympathetic regulation $(\mathrm{PR}$, heart rate $-\mathrm{HR}<80$ ) type, subgroup 1B - volunteers with balanced regulation $(\mathrm{BR}$, HR > 80) type. HRV values were measured under natural conditions, during quiet magnetic and days with GMSs. The total experiment time in both cases was 60 minutes.

The distribution of the number of volunteers in two subgroups in the cases of quiet magnetic days (QMD) and those with GMSs are shown in Table 1.

Table 1. Distribution of the volunteers during different outdoor GMA levels.

\begin{tabular}{lll}
\hline K-index & Number of volunteers group 1A-PR & Number of volunteers \\
\hline $1-3$ & 26 & 6 \\
$4-5$ & 5 & 9 \\
\hline
\end{tabular}

\subsection{Experiment 2 - Simulated Conditions}

In this stage, experiments were performed under both compensation mode (on the first day of the experiments) and GMS simulation mode (on the second day of experiments). Experiments involved $\mathrm{n}=19$ volunteers (group 2 - basic and were divided into two sub-groups: subgroup 2A (with initial PR, HR <80), subgroup 3B (with initial BR, HR > 80). The total experiment time (either for compensation or simulation regimes) was 60 minutes with three $20 \mathrm{~min}$. sub spans: (i) Detection of initial values of the ANS at rest (the magneto compensation and simulation device is turned off), Measuring of HRV under GMS compensation or GMS simulation modes, (iii) Measuring during the restoration (RS) (the magneto compensation and simulation device is turned off).

HRV measurements under the GMS compensation mode $(\mathrm{K}=0)$ correspond to a magnetic field strength within the of $(\mathrm{B}=0-5 \mathrm{nT})$ and simulation mode $(\mathrm{K}=7)$ to a natural magnetic field strength within the range of magnetic $(B=200 n T)$. In total 116 short-term $(1 \mathrm{~h})$ recordings were done. For statistical analysis of the data obtained the "Primer of Biostatistics" software by Stanton A. Glantz (fifth edition) was used with corresponded $95 \%$ CI. In total measurements lasted 4 months.

\section{Results}

\subsection{Results of the Experiment 1}

Values of HRV indices in the cases of QMD and those with GMSs are shown in Table 2.

Table 2. Values of HRV in subgroups during $Q M D$ and days with GMSs.

\begin{tabular}{|c|c|c|c|c|c|c|}
\hline Sub groups & $K$-index & HR (b/m) & SDNN (ms) & RMSSD (ms) & LF/HF (ms2/ms2) & SI (con. unit) \\
\hline PR & $1-3$ & $70.77 \pm 6.25$ & $73.9 \pm 25.62$ & $49.87 \pm 18.23$ & $1.265 \pm 0.448$ & $395.1 \pm 304.7$ \\
\hline \multirow[t]{3}{*}{ PR } & $4-5$ & $67.78 \pm 11.08$ & $76.33 \pm 26.6$ & $45.56 \pm 12.93$ & $1.561 \pm 0.374$ & $1145 \pm 926.5^{*}$ \\
\hline & $\mathrm{t}$ & 1.041 & 0.248 & 0.659 & 1.798 & 3.882 \\
\hline & $\mathrm{p}$ & 0.305 & 0.806 & 0.514 & 0.080 & $0.000 *$ \\
\hline $\mathrm{BR}$ & $1-3$ & $84.25 \pm 5.75$ & $49.38 \pm 10.18$ & $25.5 \pm 8$ & $1.529 \pm 0.371$ & $594.4 \pm 350.2$ \\
\hline \multirow[t]{3}{*}{$\mathrm{BR}$} & $4-5$ & $84.65 \pm 3.673$ & $48.65 \pm 11.83$ & $25.29 \pm 8.608$ & $1.893 \pm 0.245^{*}$ & $1704 \pm 1431^{*}$ \\
\hline & $\mathrm{t}$ & 0.21 & 0.15 & 0.057 & 2.928 & 2.14 \\
\hline & $\mathrm{p}$ & 0.836 & 0.882 & 0.955 & $0.008 *$ & $0.043 *$ \\
\hline
\end{tabular}

Remarks:

* indicates statistically significant differences.

HR - heart rate, heart beats in min.;

SDNN - Standard deviation of all Normal to Normal RR intervals, stands for the total effect of autonomic regulation of blood circulation;

RMSSD - The square root of the arithmetical mean of the sum of the squares of differences between adjacent NN intervals, stands for the activity of the PP of the ANS;

$\mathrm{LF} / \mathrm{HF}$ - Ratio LF [ms2]/HF [ms2], physiological interpretation-the ANS sympatho-vagal balance index;

$\mathrm{SI}\left(\mathrm{SI}=\mathrm{A}_{\mathrm{Mo}} / \Delta \mathrm{X} \cdot \mathrm{M}_{\mathrm{o}}\right.$; where: Mo is the mode of individual $\mathrm{R}-\mathrm{R}$ intervals, $\mathrm{A}_{\mathrm{Mo}}$ is the percentage of cycles, corresponding to Mo, and $\Delta \mathrm{X}$ is the difference between the shortest and longest R-R intervals in seconds) - The Stress Index which reflects a degree of the prevalence of central regulatory mechanisms activity over that of autonomic and characterizes, basically, activity of the SP of ANS.

Statistically significant differences between SI are observed in the two subgroups, LF/HF also shifts in the two subgroups, but statistically significant shift is observed in the subgroup 1B. These results show that an intensification of the SP of the ANS during GMSs occurred in the examined Subgroups 1A and 1B compared to the initial values (during quiet days) obtained in subgroups.

In Figures 1, 2, 3 the trends for increase of SI and LF/HF 
indices are shown in the case of initially PR and BR types on

$(\mathrm{K}=1-3)$ across the study period.

the days with GMSs $(K \geq 5)$ in comparison with the QMD

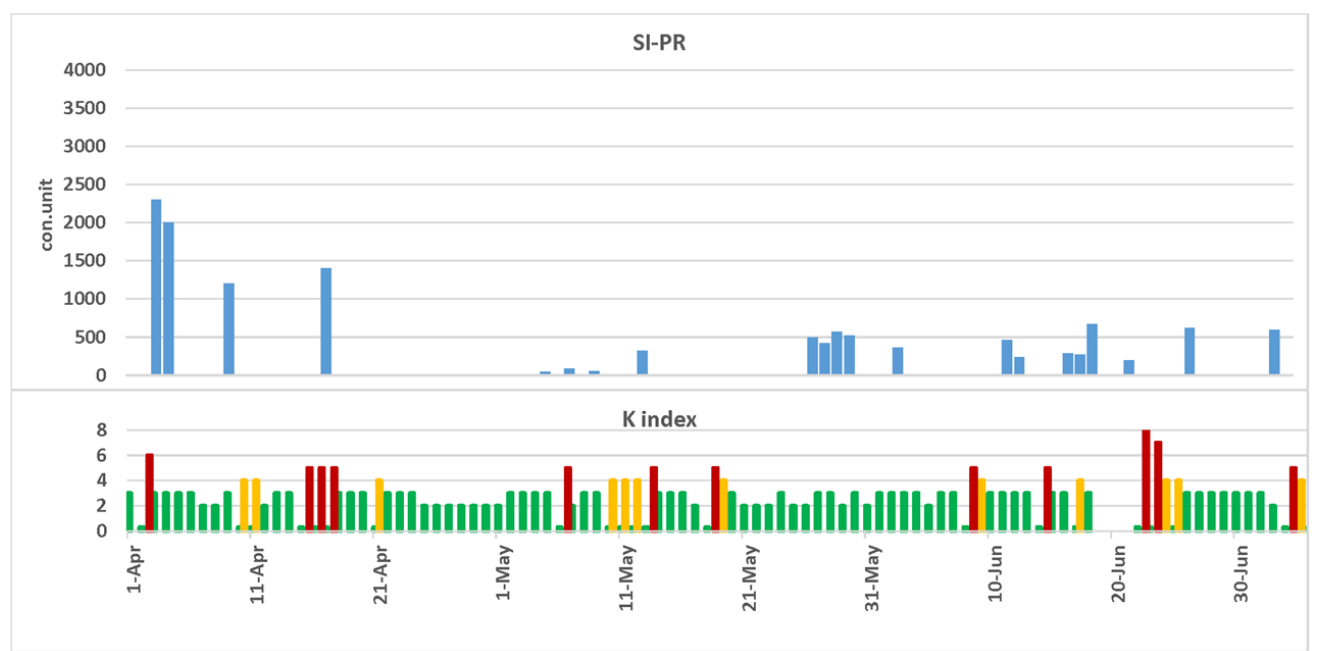

Figure 1. The trend for increase of SI on the days with GMS in the case of initially PR type (March-June 2015).

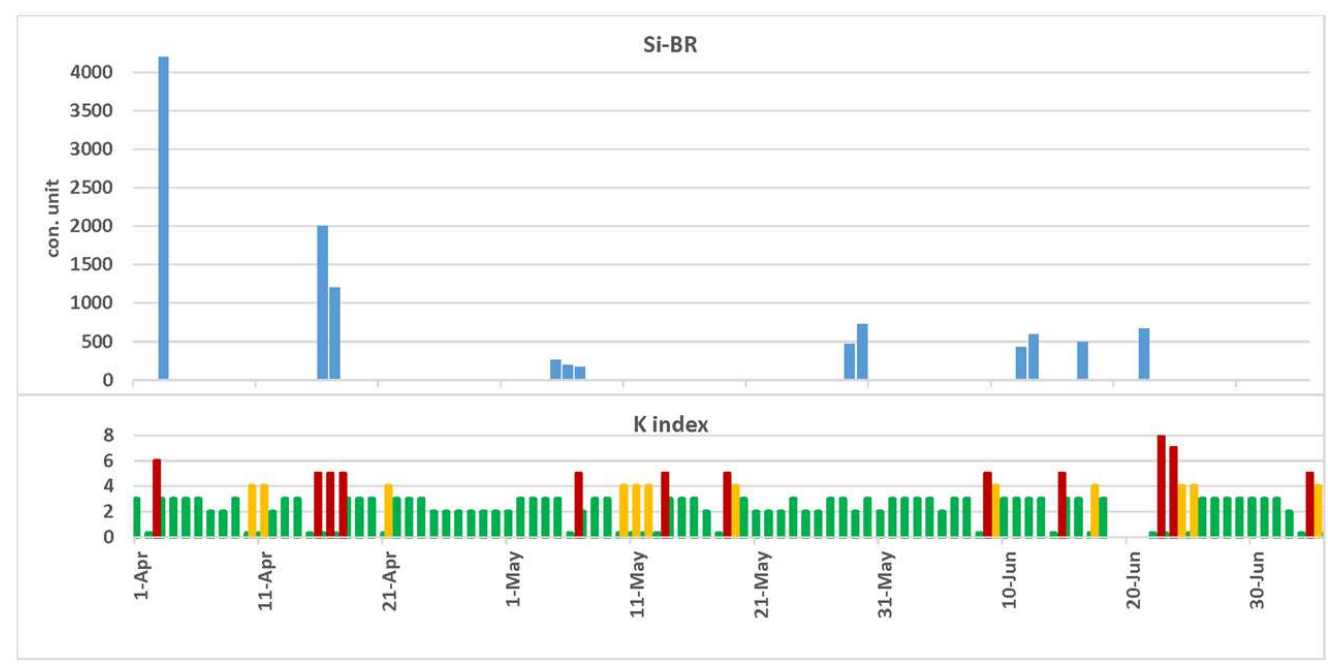

Figure 2. The trend for increase of SI on the days with GMS in the case of initially BR type (March - June 2015).

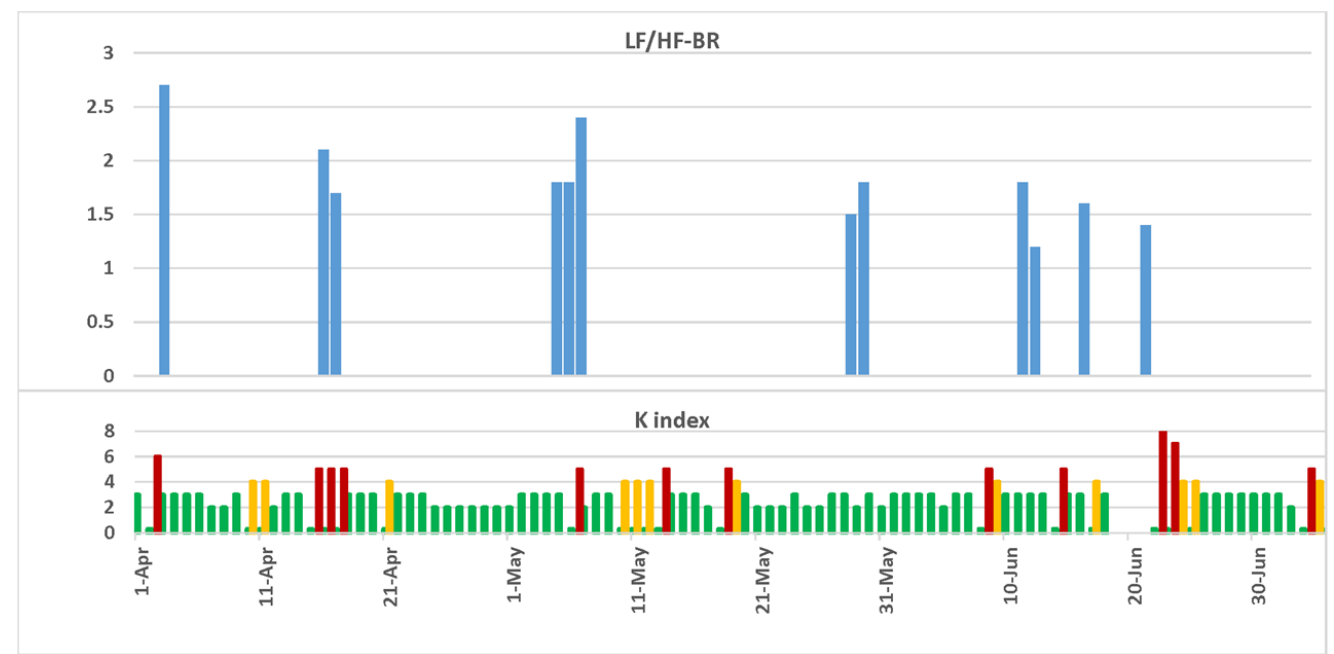

Figure 3. The trend for increase of LF/HF index on the days with GMS in the case of initially BR type (March-June 2015).

\subsection{Results of the Experiment 2}


Values of HRV under both GMS compensation and simulation modes are shown in table 3.

Table 3. Values of HRV under GMS compensation and simulation modes.

\begin{tabular}{llllllll}
\hline Subgroups & Modes & K & HR (b/m) & SDNN (ms) & RMSSD (ms) & LF/HF & SI (con. unit) \\
\hline PR & CM & 0 & $68.33 \pm 4.96$ & 99.67 & $57.33 \pm 31.3$ & $222 \pm 74$ \\
12 & SM & 7 & $69.75 \pm 5.36$ & 96.42 & $54.0 \pm 27.92$ & $1.28 \pm 0.32$ \\
t & & & 0.786 & 0.419 & 0.358 & $1.2 \pm 0.27$ \\
p & & & 0.449 & 0.683 & 0.727 & 1.33 \\
BR & CM & 0 & $76.7 \pm 4.03$ & $67 \pm 14.48$ & $38.43 \pm 11.77$ & 0.21 \\
7 & SM & 7 & $87.57 \pm 4.99^{*}$ & $54.7 \pm 10.1^{*}$ & $19.14 \pm 4.88^{*}$ & $1.1 \pm 0.14$ \\
t & & & 6.62 & 2.49 & 5.538 & $1.43 \pm 0.34^{*}$ & 2.8 \\
p & & & 0.000 & 0.047 & 0.001 & $414 \pm 69^{*}$ & 5.2 \\
\hline
\end{tabular}

\section{Remarks:}

CM - GMS compensation mode;

SM - GMS simulation mode.

As presented in Table 3, during the experiments statistically significant differences between the HRV indices were observed. A comparison of HRV indices between GMS compensation and simulation modes clearly reveals the presence of magneto sensitive reactions among those investigated:

In the case of initially PR type of volunteers exposure to the simulated GMS in the room results in a little shift to the prevalence of the PP of ANS, observable in further changes from the measured indices: SDNN, LF/HF and RMSSD reduced, while $\mathrm{HR}$ and $\mathrm{SI}$ increased that is interpreted as the prevalence of the SP of ANS, however, these changes are not statistically significant and therefore could not indicate an increased stress level.

In the case of initially BR - statistically significant intensification of the SP, as well as PP of ANS (increased HR, LF/HF and SI with reduced SDNN and RMSSD) after the exposure to the simulated GMS, has been observed that indicates a pronounced stress level.

In Figure 4 the statistically significant changes of the averages of HR (a), SDNN (b), RMSDD (c), LF/HF (d) and SI (e) in GMS simulation mode are shown in comparison with GMS compensation mode in the case of BR type of ANS in volunteers.

As shown by the results of these experiments on the impact of different GMA, more actively reacted volunteers with initial BR type compared with PR, that could be explained by the high regulatory capacity and ability for adaptation to environmental changes [28], in volunteers having BR type compared with PR type.

Based on the analysis of the data received it was determined that some indices of HRV are sensitive to specific levels of GMA. These indices include as time domain (HR, SDNN, RMSSD) as well as frequency domain ( $\mathrm{LF} / \mathrm{HF}$ ratio) components of HRV and the Stress Index.

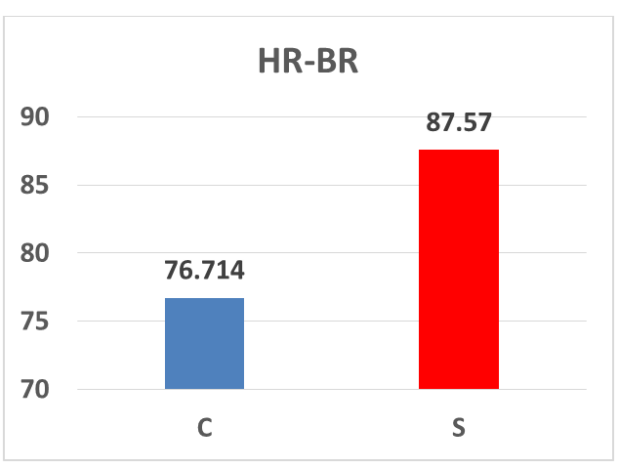

(a)

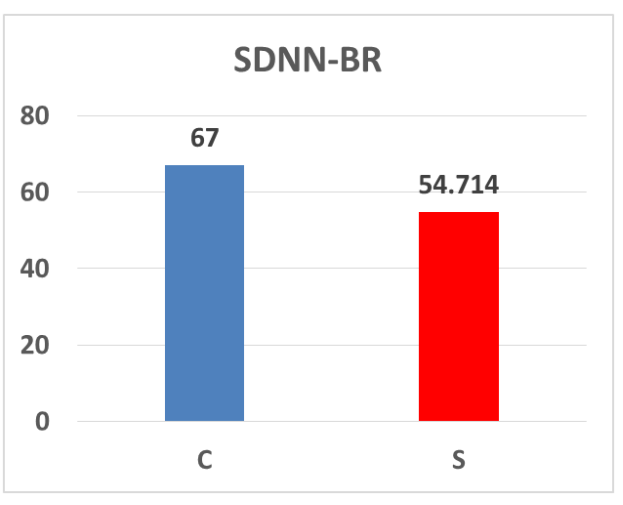

(b)

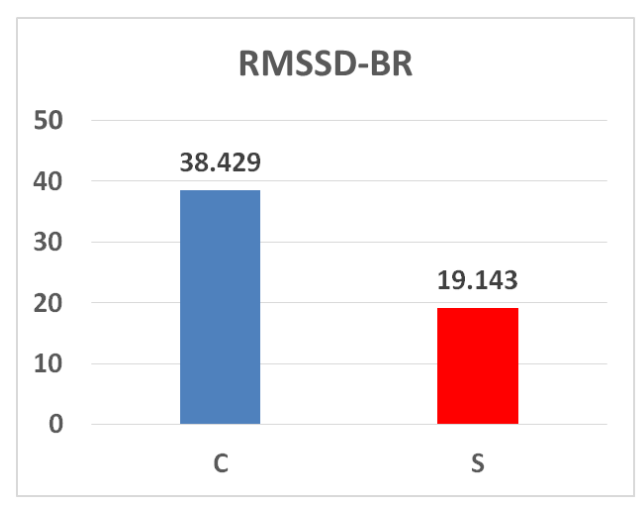

(c) 


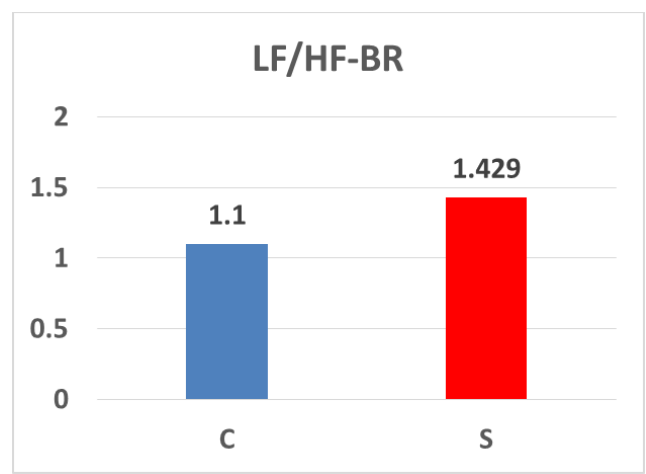

(d)

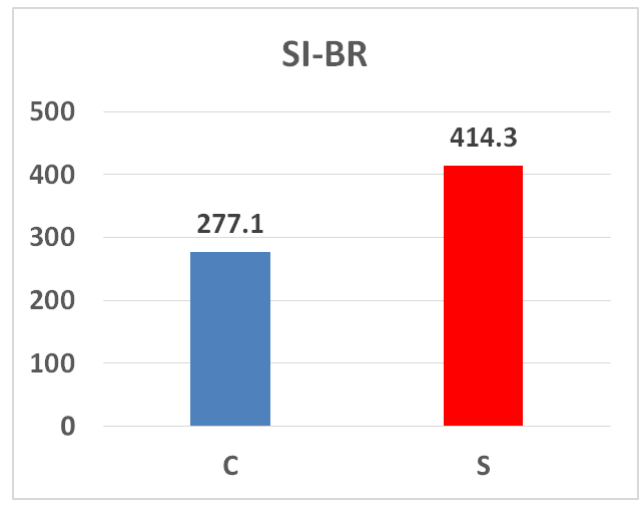

(e)

Figure 4. Comparison of the averages of $H R, S D N N, R M S D D, L F / H F$ and $S I$ in GMS compensation (c) and simulation (s) modes in the case of initially $B R$ type of ANS.

\section{Discussion}

Results of the experiments confirm the concept of Baevsky et al. [36] concerning the mechanisms of regulation of the heart rhythm, namely that PP and SP co-operate in a specific way and both branches are affected by some humoral and reflex factors and the central nervous system. There is a permanent effect of sympathetic and parasympathetic influences at all levels of the regulation. The true relations between the two parts of the ANS are complex. The optimal adaptive reaction would be achieved by the decrease of activity in one part of the ANS and increase in another.

Results of the experiments confirm the hypothesis of Berntson et al. [28], that the PP and SP competitively regulate HR (accentuated antagonism), where increased sympathetic activity is paired with decreased parasympathetic activity and that both branches of the ANS are simultaneously active. These results confirm those found by several authors from section 1 [20], who observed the increased HRV related to the different initial states of ANS regulation during days with high GMA. Furthermore, the obtained results extend some previous studies [17-19], that report finding a negative correlation between HRV and the level of GMA which supports the idea that the reduction in HRV could be related to the response of the ANS to changes in GMA (GMF levels), that can be either sympathetic or parasympathetic in a particular individual.

Results are found to partly confirm the results by Billman [27], that the LF/HF ratio does not accurately measure the cardiac sympathovagal balance; Results of the experiments have followed the concepts that the actual heart rhythm reflects not only the simple sum of sympathetic and parasympathetic activity but also that the current activity is the system reaction of a multilevel system of regulation [36] and reflect both physiological and psychological functional status of internal self-regulatory systems [34]. Results extend those found by Chernouss et al. [21], who determined that the following three HRV indices are the most sensitive to the GMA impact: the LF, HF spectra power, and the SI.

\section{Conclusions}

The performed experimental studies consistently showed that GMA events produce significant health effects. Measurements carried out under either natural or artificially simulated GMS conditions were found to coincide with each other. In comparison with quiet magnetic days $(\mathrm{K}=1-3)$, the initial values of the HRV indices significantly shifted towards intensification of the SP of ANS during days of GMSs $(\mathrm{K}=5-7)$. The ANS balance in healthy subjects was observed to shift during direct short-term exposure to simulated GMSs with intensities in the range of natural GMSs $(\mathrm{K}=7, \mathrm{~B}=200 \mathrm{nT})$ in comparison with conditions during compensated GMSs $(\mathrm{K}=0, \mathrm{~B}=0-5 \mathrm{nT})$.

Interpretations of the received results are summarized here:

GMSs have an effect on healthy humans at middle latitudes.

High values of GMA have a sensitive effect on humans causing specific stress-reactions.

Changes in GMA are correlated with HRV that is related to the ANS response in a particular individual.

ANS response to the exposure to natural or simulated GMSs showed an intensification of both part of the ANS, though initial types of ANS regulation resulted in different dynamics of alterations during different GMA levels.

Seems healthy humans with initial BR type of the ANS have more regulatory capacity for adaptation to the changes on GMA, compared with PR type.

These conclusions give support for the hypothesis that GMF as an unusually weak magnetic field when disturbed can have effects on human health. The efficacy of the applied abovementioned methodology confirmed by the results from these experiments to evaluate humans' sensitive reactions to GMA. Our study has some limitations due to the number of subjects in the various groups being small. We propose to continue such experimenting, by collecting and analyzing more data and using the device for GMS compensation and simulation. The advantages of the device are that they enable creating the required GMF activity at any time regardless of external GMF activity. A further analysis study to search systematically for correlations with variations of solar-induced GMA and humans' sensitive reactions would 
provide more deep results that would either support or refute the current results.

\section{Declarations}

In this work, the following ethical standards were complied with: The laws of Georgia, the Helsinki Declaration as well as data protection stipulations. The written consent was obtained by all participants of the study. The study was conducted according to the guidelines of the clinical trial service of the U.S. National Institutes of Health (https://clinicaltrials.gov/ct2/about-studies/learn). The strengths of the simulated MF were in the range of the lower permissible MF exposure limits (0-200 nT) in "accordance" with the WHO's international Magnetic Fields Health and Safety Guides: "IPCS International Program on chemical Safety, Health and Safety Guide No. 27, Magnetic Fields Health and Safety Guide (http://www.inchem.org/documents/hsg/hsg/hsg027.htm).

\section{Availability of Data and Materials}

The detailed datasets used for analyzing during the current study are available from the corresponding author on reasonable request.

\section{Competing Interests}

The authors declare that they have no competing interests.

\section{Funding}

This work was supported by the International Science and Technology Center (ISTC) (grant \#G-2094).

\section{Authors Contribution Statement}

Ketevan Janashia wrote the main text of the manuscript and analyzed the results, Levan Tvildiani and Tamar Tsibadze led the medical experiments, Nikoloz Invia created magnetocompensation/simulation device and simulated GMF, Vasili Kukhianidze collected GMSs and satellite data, George Ramishvili processed the received data and designed the manuscript.

All authors reviewed the manuscript.

\section{Acknowledgements}

The authors thank Dr. Norma B. Crosby from Royal Belgian Institute for Space Aeronomy for actively collaborating within the project.

\section{References}

[1] Binhi V. Stochastic dynamics of magnetosomes a mechanism of biological orientation in the geomagnetic field. Bioelectromagnetics, 2006, 27 (1): 58-63.

[2] Binhi V. Do naturally occurring magnetic nanoparticles in the human body mediate increased risk of childhood leukaemia with EMF exposure? Intern. J. Radiation Biology, 2008, 84 (7): 569-579.

[3] Cherry N. Schumann Resonances, a plausible biophysical mechanism for the human health effects of Solar/Geomagnetic Activity. Natural Hazards, 2002, 26, 279-331.

[4] Carrubba S, Frilot II C, Chesson Jr AL, Marino AA. Evidence of a nonlinear human magnetic sense. Neuroscience, 2007, 144, 356-367.

[5] Hart F. A Quantum Mechanical Model for Bioelectromagnetic Resonance Phenomena. Electromagnetic Biology and Medicine, 2010, 9 (1): 1-7.

[6] Brown F. Biological clocks Endogenous cycles synchronized by subtle geophysical rhythms bio systems. Biosystems, 1976, 8 (2): $67-81$.

[7] Ulmer W, Cornélissen G. Coupled Electromagnetic Circuits and Their Connection to Quantum Mechanical Resonance Interactions and Biorhythms. Open Journal of Biophysics, 2013, 3 (4): 253-274.

[8] Zenchenko TA, Medvedeva AA, Khorseva NI, Breus TK. Synchronization of Human Heart Rate Indicators and Geomagnetic Field Variations in the Frequency Range of 0.5$3.0 \mathrm{mHz}$. Izvestiya, Atmospheric and Oceanic Physic, 2014, 50 (7): 736-744.

[9] Malmivuo J, Plonsey R. Bioelectromagnetism: Principles and applications of bioelectric and biomagnetic fields. 1995, Oxford: Oxford Univ. Press, England.

[10] Khabarova O, Dimitrova S. On the nature of people's reaction to space weather and meteorological weather changes. Sun and Geosphere, 2009, 4 (2): 60-71.

[11] McCraty R, Atkinson M, Stolc V, et al. Synchronization of Human Autonomic Nervous System Rhythms with Geomagnetic Activity in Human Subjects. Int. J. Environ. Res. Public Health, 2017, 13; 14 (7): 770.

[12] Wood AW, Armstrong SM, Sait M, L. et al. Changes in human plasma melatonin profiles in response to $50 \mathrm{~Hz}$ magnetic field exposure. Pineal research, 2007, 25 (2): 116-127.

[13] Halberg F, Cornelissen G, McCraty R, Al-Abdulgader A. Time structures (chronomes) of the blood circulation, populations' health, human affairs and space weather. World Heart J, 2011, $3(1): 1-42$.

[14] Palmer SJ, Rycroft MJ, Cermack M. Solar and geomagnetic activity, extremely low frequency magnetic and electric fields and human health at the Earth's surface. Surv Geophys, 2006, 27 (5): 557-595.

[15] Khorseva N. Using psychophysiological indices to estimate the effect of cosmophysical factors (review). Izv. Atmos. Ocean. Phys, 2013, 49 (8): 839-852.

[16] Vencloviene V, Babarskiene R, Kaminskaite B, Vasiliauskas D. The Effect of Solar-Geomagnetic Activity During Hospital Admission on the Prognosis of Cardiovascular Outcomes in Patients with Myocardial Infarction. British Journal of Medicine and Medical Research, 2013, 3 (4): 1587-1597.

[17] Otsuka K, Cornelissen G, Weydahl A, et al. Geomagnetic disturbance associated with decrease in heart rate variability in a subarctic area. Biomed. Pharmacother, 2001, 55 (S1): 5156. 
[18] Gmitrov J, Ohkubo C. Geomagnetic field decreases cardiovascular variability. Electromagn. Magnetobiology, 1999, 18: 291-303.

[19] Breus T, Baevsky R, Chernikova A. Effects of geomagnetic disturbances on humans' functional state in space flight. J. Biomed. Sci. Eng, 2012, 5: 341-355.

[20] Al-Abdulgader A, McCraty R, Atkinson M, et al. Long-Term Study of Heart Rate Variability Responses to Changes in the Solar and Geomagnetic Environment. Scientific Reports, 2018, 8: 2663

[21] Chernouss S, Vinogradov A, Vlassova E. Geophysical hazard for human health in the Circumpolar Auroral Belt: evidence of a relationship between heart rate variation and electromagnetic disturbances. Natural Hazards, 2001, 23 (2-3): 121-135.

[22] Kay RW. Geomagnetic storms: association with incidence of depression as measured by hospital admission. Br. J. Psychiatry, 1994, 164: 403-409.

[23] Shaffer F, McCraty R, Zerr C. A healthy heart is not a metronome: An integrative review of the heart's anatomy and heart rate variability. Front. Psychol, 2014, 5: 1040.

[24] Pagani M, Lombardi F, Guzzeti S, et al. Power spectral analysis of heart rate and arterial pressure variabilities as a marker of sympatho-vagal interaction in man and concious dog. Circulation Research, 1986, 59: 178-193.

[25] Malliani A, Pagani M, Lombardi F, S. Cerutti S. Cardiovascular neural regulation explored in the frequency domain. Circulation, 1991, 84: 482-492.

[26] Eckberg D. Sympathovagal Balance: A Critical Appraisal. Circulation, 1997, 96 (9): 3224-32.

[27] Billman G. The LF/HF ratio does not accurately measure cardiac sympatho-vagal balance. Frontiers in Physiology, 2013, 4: 26.
[28] Bernston G, Bigger JJ, Eckberg D, et al. Heart rate variability: origins, methods and interpretive caveats. Psychophysiology, 2007, 34 (1997): 623-648.

[29] Menvielle M, Iyemori T, Marchaudon A, Nose M. Geomagnetic indices, $\mathrm{K}$ index limits, in "Geomagnetic Observations and Models", by M. Mandea and M. Korte (Eds), IAGA Special Sopron Book Series, 2011, 201.

[30] Invia N, Kavlashvili N, Kubaneishvili E, The system of compensation and simulation of perturbed geomagnetic field variations, Works of Archil Eliashvili Institute of Control System of the Georgian Technical University, 2015, 19: 39-43.

[31] Janashia K, Tvildiani L, Tsibadze T, Invia N. et al. Effects of magnetoactive compensation of geomagnetic field on heart rate variability of healthy males. J. Sun and Geosphere, 15 (1) in press.

[32] Tvildiani L, Janashia K, Tsibadze A, Invia N, Kubaneishvili E. The technique of the simulation of geomagnetic storms. Georgian Patent 6823, 15 March, 2018.

[33] European Society of Cardiology and the North American Society of Pacing and Electrophysiology, Heart Rate Variability, 1996, European Heart Journal, 17: 354-381.

[34] McCraty R, Shaffer F, Heart rate Variability: new perspectives on physiological Mechanisms, assessment of self-regulatory Capacity, and Health risk. Global advances in health and medicine, 2015, 4 (1): 46-61.

[35] Baevsky R, Berseneva A. Methodical recommendations: Use KARDiVAR System for determination of the stress level and estimation of the body adaptability, Standards of measurements and physiological interpretation, 2008, http://www.ehrlich.tv/Kardivar_Methodical_Eng.pdf.

[36] Bayevsky R, Ivanov G, Chireyikin L, et al. HRV Analysis under the usage of different electrocardiography systems. 2002, Commitee of Clinic Diagnostic apparatus and the Commitee of New Medical Techniques, Moskow. 